\title{
Is Single-Radius Design Better for Quadriceps Recovery in Total Knee Arthroplasty?
}

\author{
Duk-Hyun Kim, $\mathrm{MD}^{1}$, Dong-Kyoon Kim, $\mathrm{MD}^{2}$, Sang-Hak Lee, $\mathrm{MD}^{1}$, Kang-Il Kim, MD ${ }^{1}$, and \\ Dae-Kyung Bae, $\mathrm{MD}^{2}$ \\ ${ }^{1}$ Department of Orthopaedic Surgery, Center for Joint Diseases and Rheumatism, Kyung Hee University Hospital at Gangdong, Seoul; ${ }^{2}$ Department of Orthopaedic \\ Surgery, Kyung Hee University Hospital, Seoul, Korea
}

\begin{abstract}
Purpose: Although single-radius (SR) designs have a theoretical advantage in quadriceps recovery following total knee arthroplasty (TKA), there has been a paucity of objective evaluation studies.

Materials and Methods: One hundred and twenty minimally invasive TKAs were prospectively randomized by a single surgeon into 2 groups: SR design TKA group and multi-radius design TKA group. Quadriceps force and power were assessed using a dynamometer, and clinical data were investigated preoperatively and 6 weeks, 3 months, 6 months and 1 year postoperatively.

Results: There were no differences between two groups in quadriceps recovery and clinical results throughout the follow-up period. Furthermore, the proportion of patients whose postoperative quadriceps force and power reached preoperative level was similar in both groups.

Conclusions: Femoral component design itself would not significantly influence quadriceps recovery after TKA.
\end{abstract}

Keywords: Knee, Arthroplasty, Prosthesis design, Quadriceps muscle, Muscle strength dynamometer

\section{Introduction}

Adequate function of the extensor mechanism after total knee arthroplasty (TKA) is essential for a successful clinical outcome as well as participation in daily living activities ${ }^{1,2)}$. Quadriceps strength is the major determinant of extensor mechanism function, which is affected by a variety of factors in TKA. Singleradius (SR) and multi-radius (MR) femoral designs are believed to have different levels of influence on the recovery of quadriceps muscle strength ${ }^{3-5)}$. SR implants are designed to have a more posterior center of rotation, thus increasing the moment arm of

Received June 10, 2015; Revised September 21, 2015;

Accepted October 19, 2015

Correspondence to: Kang-Il Kim, MD

Department of Orthopaedic Surgery, Center for Joint Diseases and

Rheumatism, Kyung Hee University Hospital at Gangdong, 892

Dongnam-ro, Gangdong-gu, Seoul 05278, Korea

Tel: +82-2-440-6151, Fax: +82-2-440-6296

E-mail: drkim@khu.ac.kr

This is an Open Access article distributed under the terms of the Creative Commons Attribution Non-Commercial License (http://creativecommons.org/licenses/by-nc/4.0/) which permits unrestricted non-commercial use, distribution, and reproduction in any medium, provided the original work is properly cited. the patellar tendon, requiring less quadriceps force, and lessening the load on the patella) ${ }^{6}$ as demonstrated in cadaver studies ${ }^{3,4,7,8)}$. Furthermore, the SR design has a theoretical advantage of minimizing ligament instability during mid-flexion based on maintenance of ligament isometry through the entire range of motion $(\mathrm{ROM})^{9,10)}$.

Few studies have extensively compared the SR and MR femoral components, and most of which focused on the clinical scores and $\mathrm{ROM}^{11-14)}$. It is difficult to find studies that include preoperative or sequential follow-up data on quadriceps strength assessed in an objective manner ${ }^{12)}$. The purpose of the current study was to determine whether the theoretical advantage of the SR femoral design with regard to quadriceps recovery could be realized in a clinical setting.

Based on the theoretical advantage of the SR design documented in published research, we derived and tested the following hypotheses: 1) the quadriceps force and power values would be higher in the SR femoral design group; 2) the proportion of patients with physiological levels of quadriceps force and power would be higher in the SR group; and moreover we compared the clinical outcome based on the American Knee Society score (AKSS) between two groups. 


\section{Materials and Methods}

A total of 164 patients (164 knees) who underwent elective primary TKA were initially enrolled in the study. The indication for surgery was degenerative osteoarthritis in all patients. In order to rule out the influence of gender difference in muscle strength on the results, the study population was composed of female only. The exclusion criteria were the presence of following conditions: 1) $\geq 30^{\circ}$ of flexion contracture; 2) $<100^{\circ}$ of ROM arc; 3) valgus deformity; 4) diagnosis other than primary osteoarthritis such as inflammatory arthritis, haemophilic arthropathy, or posttraumatic arthritis; and 5) a history of cerebrovascular accidents or presence of knee pain restricting ambulation or decreasing muscle strength of the lower limb. After excluding 44 knees based on the above-listed criteria (Fig. 1), we evaluated the preoperative and postoperative clinical data of 120 consecutive TKAs performed using a midvastus approach. The 120 patients were assigned using a computer-generated randomization table into the SR group and the MR group. Sample size was calculated from a pilot study aimed at determining whether the quadriceps force (primary outcome) is significantly higher in the SR group at 1 year after surgery. The required sample size was calculated as 108 (54 per each group) assuming a two-sided type I error rate of 0.05 and a power of 0.80 . Ultimately, 120 patients were included in the study to allow for a $10 \%$ attrition rate at 12 months after surgery. This study was conducted with Institutional Review Board approval and informed consent from all patients.

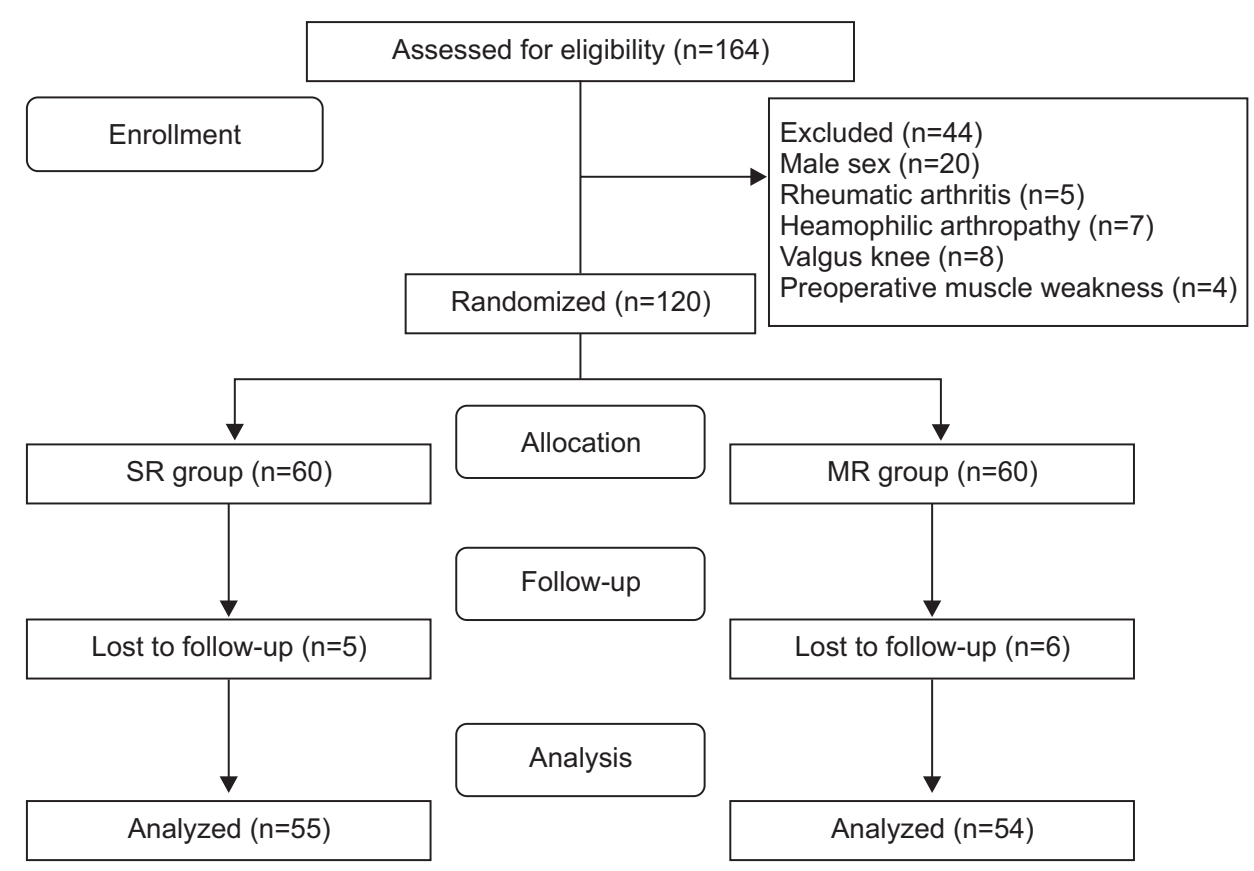

The surgery was performed by the same surgeon (Kim KI) via a minimally invasive approach in all patients. The SR group received the Triathlon (Stryker Orthopaedics, Mahwah, NJ, USA) and the MR group received the PFC Sigma (DePuy, Warsaw, IN, USA). PFC Sigma is designed with several axes of rotation, the so called MR design: the axes make J-curve and have various radius sizes not defined as single range. Triathlon is designed with single axis of rotation: the center of axis is about the transepicondylar axis and the single size of radius ranges from $10^{\circ}$ to $110^{\circ}$. In order to eliminate any influence of difference between the cruciate retention component and posterior stabilizing component, the implant was a posterior stabilized design in all patients. Bonecement (Simplex P, Stryker Orthopaedics) was used for fixation in all knees. In both groups, patella resurfacing was performed if the patient was over 60 years of age and presented with $\geq$ grade III arthritis, apparent anterior knee pain, and a patellar thickness of $\geq 20 \mathrm{~mm}$. General anesthesia was used during surgery, and patient-controlled analgesia and intraoperative periarticular injection were administered for pain control. The postoperative rehabilitation regimen was identical for both groups.

In order to obtain objective numeric data, we assessed the extent of quadriceps recovery using a dynamometer at the same time points until the first postoperative year and compared the values obtained from the SR group and MR group for analysis.

For quantification of the extent of quadriceps recovery, quadriceps strength was assessed using the Baltimore Therapeutic Equipment Primus (BTE, Hanover, MD, USA) preoperatively
Fig. 1. CONSORT diagram showing the flow of participants through each stage of the trial. SR: single-radius, MR: multiradius. 
and 6 weeks, 3 months, 6 months, and 1 year postoperatively. The patient was seated on the chair of the dynamometer with the shoulder and hip tightly secured by a strap. The patient was informed about the testing procedures in advance so that the test could be conducted with the understanding and consent of the patient. The testing was performed by an independent physical therapist who did not participate in the surgery and was blinded to the group allocation. Quadriceps force was measured in Newtons and quadriceps power was measured in Watts, and the measurements were recorded by an automated system. Quadriceps force was defined as the maximum isometric effort exerted during full extension from $90^{\circ}$ of knee flexion. Quadriceps power, which refers to the amount of work per unit of time, was measured by asking the patient to maintain $60^{\circ}$ of knee flexion and extension as long as possible during a period of 10 seconds under the application of a load equal to $50 \%$ of the quadriceps force. The tests were performed three times each.

A value was accepted as valid if it was in the proximity of the other measured values; if a value deviated considerably from the other values, measurement was repeated assuming an error had occurred. To evaluate the extent of quadriceps recovery, quadriceps force and power values and the percentage of improvement compared to the preoperative value were recorded and used for analysis.

Preoperatively, weight-bearing anteroposterior, lateral, and $45^{\circ}$ flexion radiographs of both knees, Merchant views, and standing long leg radiographs were obtained in all patients. At 6 days, 6 weeks, 3 months, 6 months, and 12 months after surgery, anteroposterior and lateral radiographs and long leg standing radiographs were acquired. The ROM and AKSS knee/function score were assessed preoperatively and at each follow-up session.

All statistical analyses were performed using the SPSS ver. 16.0 (SPSS Inc., Chicago, IL, USA). The quadriceps force, AKSS (knee score and function score), and ROM were compared between the groups using independent sample t-tests. A chi-square test was used for comparison of the extent of recovery of the quadriceps force and power relative to the preoperative level at each followup session between the groups. For comparison of changes between the preoperative and follow-up quadriceps force values, a repeated measures analysis of variance was conducted. A p-value of less than 0.05 was considered statistically significant.

\section{Results}

Ultimately, there were 55 patients in the SR group and 54 patients in the MR group. There was no significant intergroup difference with regard to patient demographics (Table 1). The

Table 1. Patient Demographics

\begin{tabular}{lccc}
\hline \multicolumn{1}{c}{ Parameter } & $\begin{array}{c}\text { SR group } \\
(\mathrm{n}=55)\end{array}$ & $\begin{array}{c}\text { MR group } \\
(\mathrm{n}=54)\end{array}$ & p-value \\
\hline Age $(\mathrm{yr})$ & $67.2 \pm 6.2$ & $67.2 \pm 8.3$ & $>0.05$ \\
Body mass index $\left(\mathrm{kg} / \mathrm{m}^{2}\right)$ & $27.4 \pm 3.7$ & $27.1 \pm 2.6$ & \\
Force $(\mathrm{N})$ & $160.8 \pm 60.9$ & $148.9 \pm 67.4$ & \\
Power $(\mathrm{W})$ & $12.1 \pm 7.3$ & $10.8 \pm 7.3$ & \\
Range of motion $\left({ }^{\circ}\right)$ & $111.5 \pm 6.2$ & $112.8 \pm 6.2$ & \\
Preop further flexion $\left(^{\circ}\right)$ & $129.6 \pm 6.7$ & $128.2 \pm 14.4$ & \\
Preop AKSS knee score & $50.9 \pm 15.3$ & $45.5 \pm 20.3$ & \\
Preop AKSS function score & $49.7 \pm 12.9$ & $49.8 \pm 20.3$ & \\
Hip-knee-ankle angle $\left({ }^{\circ}\right)^{\mathrm{a})}$ & $-9.5 \pm 4.2$ & $-9.7 \pm 6.2$ & \\
Patellar resurfacing $(\%)$ & $19 / 55(35)$ & $21 / 54(39)$ & \\
\hline
\end{tabular}

Values are presented as mean \pm standard deviation.

SR: single-radius, MR: multi-radius, Preop: preoperative, AKSS: American Knee Society score.

${ }^{\text {a) }}$ Varus alignment.

Table 2. Dynamometric Data on Quadriceps Force and Power

\begin{tabular}{|c|c|c|c|c|c|c|c|c|}
\hline \multirow{3}{*}{$\begin{array}{l}\text { Parameter } \\
\text { Preop }\end{array}$} & \multicolumn{4}{|c|}{ Force in Newton } & \multicolumn{4}{|c|}{ Power in Watt } \\
\hline & \multirow{2}{*}{$\begin{array}{l}\text { SR group } \\
(n=55)\end{array}$} & \multirow{2}{*}{$\begin{array}{c}\text { MR group } \\
(\mathrm{n}=54)\end{array}$} & \multicolumn{2}{|c|}{$\mathrm{p}$-value } & \multirow{2}{*}{$\begin{array}{c}\text { SR group } \\
(n=55)\end{array}$} & \multirow{2}{*}{$\begin{array}{l}\text { MR group } \\
(\mathrm{n}=54)\end{array}$} & \multicolumn{2}{|c|}{ p-value } \\
\hline & & & $0.306^{\mathrm{a})}$ & $0.104^{\mathrm{b})}$ & & & $0.324^{\mathrm{a})}$ & $0.309^{b)}$ \\
\hline Postop $6 \mathrm{wk}$ & $112.3 \pm 41.9$ & $105.2 \pm 40.6$ & $0.409^{\mathrm{a})}$ & & $11.7 \pm 8.2$ & $10.2 \pm 6.9$ & $0.341^{\text {a) }}$ & \\
\hline Postop 3 mo & $134.8 \pm 49.1$ & $130.6 \pm 45.3$ & $0.671^{\text {a) }}$ & & $12.0 \pm 6.9$ & $11.8 \pm 6.8$ & $0.898^{\mathrm{a})}$ & \\
\hline Postop 6 mo & $176.8 \pm 69.4$ & $152.2 \pm 56.4$ & $0.167^{\mathrm{a})}$ & & $22.2 \pm 24.5$ & $20.7 \pm 29.8$ & $0.805^{\mathrm{a})}$ & \\
\hline Postop 1 yr & $206.4 \pm 74.1$ & $181.8 \pm 66.6$ & $0.108^{\mathrm{a})}$ & & $23.2 \pm 10.5$ & $19.6 \pm 11.1$ & $0.142^{\mathrm{a})}$ & \\
\hline
\end{tabular}

Values are presented as mean \pm standard deviation.

SR: single-radius, MR: multi-radius, Preop: preoperative, Postop: postoperative.

${ }^{\text {a) }}$ Independent samples t-test, ${ }^{\text {b) }}$ Repeated measures analysis of variance. 
quadriceps force and power measured preoperatively and at every follow-up session until 1 year after the surgery were not significantly different between the groups (Table 2). No notable intergroup difference was observed at each follow-up session with regard to the proportion of patients who achieved their preoperative levels of quadriceps force and power after TKA. In both groups, the mean postoperative quadriceps force at 6 months could reach the preoperative level, and the mean postoperative quadriceps power at 3 months could reach the preoperative level (Figs. 2 and 3). At 1 year after surgery, over $80 \%$ of the patients in both groups showed higher values than their preoperative quadriceps force and power (Table 3). The ROM and AKSS knee/

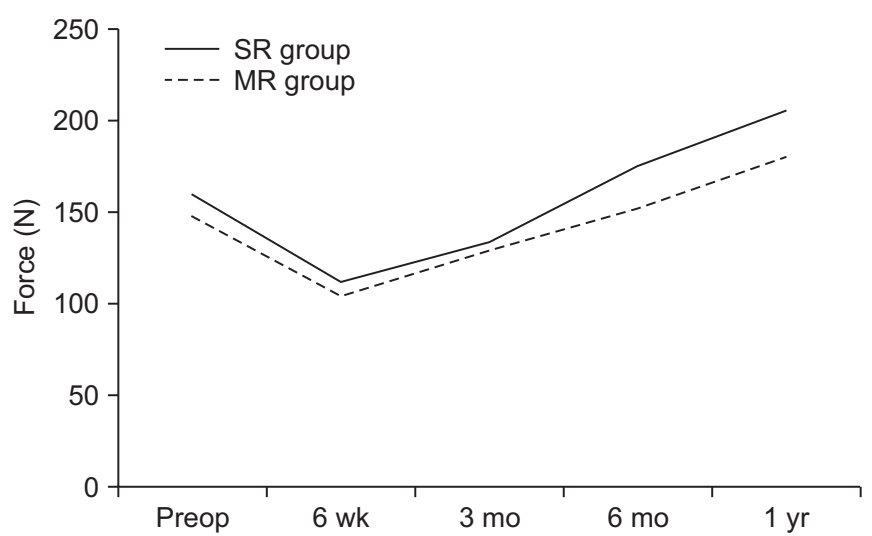

Fig. 2. Dynamometric data on isometric force. Preop: preoperative, SR: single-radius, MR: multi-radius. function score at 1 year after surgery were significantly improved, but no intergroup difference was found (Table 4).

\section{Discussion}

The principal finding of this study was there was no notable difference in quadriceps recovery related to the radius design of the femoral component. Postoperative extensor mechanism function in TKA has a considerable influence on a broad range of activities. Therefore, restoration of extensor mechanism function is essential for a favorable clinical outcome and patient satisfaction ${ }^{15)}$. Quadriceps strength that reflects extensor mechanism function

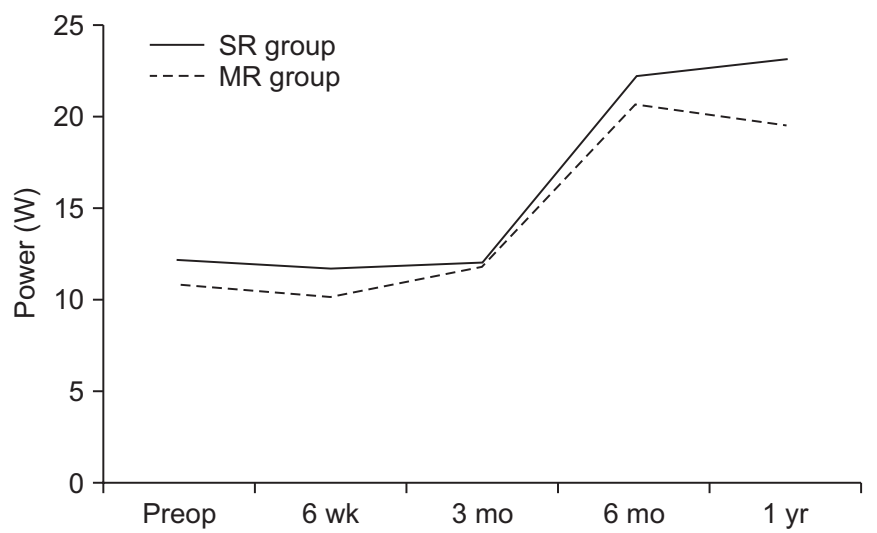

Fig. 3. Dynamometric data on isokinetic power. Preop: preoperative, SR: single-radius, MR: multi-radius.

Table 3. Rate of Recovery to or above the Preoperative Level

\begin{tabular}{|c|c|c|c|c|c|c|}
\hline \multirow{2}{*}{ Parameter } & \multicolumn{3}{|c|}{ Force in Newton } & \multicolumn{3}{|c|}{ Power in Watt } \\
\hline & SR group $(n=55)$ & MR group $(n=54)$ & $\mathrm{p}$-value & SR group $(n=55)$ & MR group $(n=54)$ & p-value \\
\hline Postop $6 \mathrm{wk}$ & $10(19)$ & $14(26)$ & $>0.05$ & $25(45)$ & $26(47)$ & $>0.05$ \\
\hline Postop 3 mo & $20(35)$ & $21(38)$ & & $28(51)$ & $37(66)$ & \\
\hline Postop 6 mo & $31(55)$ & $30(53)$ & & $39(70)$ & $41(73)$ & \\
\hline Postop 1 yr & $50(89)$ & $44(80)$ & & $50(89)$ & $50(90)$ & \\
\hline
\end{tabular}

Values are presented as number of cases (\%).

SR: single-radius, MR: multi-radius, Postop: postoperative.

Table 4. Clinical Data on American Knee Society Score and Range of Motion

\begin{tabular}{lccccccc}
\hline \multirow{2}{*}{ Parameter } & \multicolumn{3}{c}{ Preoperative } & & \multicolumn{3}{c}{ Postoperative } \\
\cline { 2 - 3 } \cline { 6 - 8 } & SR Group & MR Group & p-value & & SR Group & MR Group & p-value \\
\hline Knee score & $50.9 \pm 15.3$ & $45.5 \pm 20.3$ & $>0.05$ & & $96.9 \pm 15.3$ & $96.3 \pm 5.8$ & 90.05 \\
Functional score & $49.7 \pm 12.9$ & $49.8 \pm 20.3$ & & & $90.1 \pm 16.8$ & $92.3 \pm 8.5$ & $128.6 \pm 16.8$ \\
Range of motion $\left({ }^{\circ}\right)$ & $111.5 \pm 6.2$ & $112.8 \pm 6.2$ & & $131.4 \pm 8.0$ & \\
\hline
\end{tabular}

Values are presented as mean \pm standard deviation.

SR: single-radius, MR: multi-radius. 


\section{Kim et al. Single-Radius TKA Did Not Produce Better Quadriceps Recovery}

is regarded as a major predictor of clinical outcome of TKA, and the radius of curvature of the femoral component appears to affect quadriceps recovery. In particular, there are extremely few studies that provide quantitative data on the extent of quadriceps recovery that is considered as the primary benefit of the femoral component with a single sagittal radius ${ }^{12)}$. One study ${ }^{12)}$ attempted to demonstrate the superiority of a SR femoral design in terms of postoperative quadriceps function with quantifiable data obtained using a dynamometer. However, the study had some limitations that could compromise the validity of the results, such as the difference in the follow-up period between the SR group and the MR group. Therefore, we attempted to design a study based on more reliable and comprehensive data.

In a retrospective study comparing 30 patients with a SR design and the equivalent number of patients with a MR design, the SR group exhibited higher extension peak torque and flexion/ extension ratio $^{12)}$. Considering that the last parameter best reflects quadriceps function, the study suggests that the SR design is superior in terms of short-term functional outcome. However, their preoperative values and sequential follow-up data were not available in the study. The analysis of the study was based solely on the final follow-up data. Thus, it was difficult to determine whether there was no inherent preoperative difference in muscle strength between the groups. In addition, the final follow-up assessment was performed later in the SR group. Wang et al. ${ }^{16)}$ analyzed the electromyogram of various muscles during a sit-tostand movement. They noted that the SR design facilitated more effective performance than the MR design. However, there were differences in age and follow-up periods between the groups, and the number of cases was relatively small (8 in each group). On the other hand, Hall et al. ${ }^{13)}$ reported there was no statistically significant difference between the sing-radius group and the MR group with regard to quadriceps function that was evaluated, without using a dynamometer though, based on the ability to rise from a chair without assistance. In the current study, the objective quadriceps force and power assessed using a dynamometer were not significantly higher in the knees with a SR femoral component than those with a MR femoral component at each followup session until 1 year after TKA. Moreover, the proportions of patients with the postoperative quadriceps force and power the same or even beyond the preoperative level were not different between the groups. These findings may support the above study and femoral component design itself has no significant influence on the quadriceps recovery after TKA.

Clinical outcomes of TKA using SR femoral components have been published in previous studies. In a 3.9-year follow-up study comparing 426 knees with a SR design and 133 knees with a MR design, the former group obtained more satisfying results in terms of pain, stability knee flexion, stair climbing, clutching walking, and AKKS knee/function score ${ }^{11)}$. However, the number of subjects was not equivalent between the groups and there was a follow-up loss of $20 \%$. Jo et al. ${ }^{17)}$ described there was no significant difference with regard to the Hospital for Special Surgery scores and WOMAC (Western Ontario and McMaster Universities) scores between the SR femoral design group and the MR femoral design group. These studies indicate there is still no consensus on the relationship between the radius of curvature of the femoral component and the clinical outcome of TKA. In our cohorts, we could not observe intergroup difference with regard to the AKSS knee/function score and ROM. It might be reasonable to surmise that clinical outcome of TKA is not solely dependent on the difference of radius of the femoral component but on a variety of factors such as preoperative education, pre-emptive analgesia, local infiltration anesthesia, minimally invasive technique, and postoperative rehabilitation ${ }^{18,19)}$.

There are conflicting reports on the recovery of quadriceps after TKA compared to the preoperative status. Some studies showed that quadriceps strength after TKA was less than or rarely recovered to the normal level ${ }^{20,21)}$. They attributed this to persistent muscle weakness, surgical trauma during TKA, and age-related muscle recovery dysfunction. On the contrary, other studies demonstrated substantial improvement compared to the preoperative condition $^{22,23)}$. In the current study, the mean quadriceps force and power assessed using a dynamometer at 1 year after surgery were improved compared to the preoperative status in both groups, and most of the patients achieved preoperative levels of quadriceps strength. In both groups, the mean quadriceps force at 6 months postoperative and the mean quadriceps power at 3 months postoperative were higher than the preoperative mean values. We believe it could be achieved by a complex interaction of minimally invasive technique using a mini mid-vastus approach and early rehabilitation protocol through multimodal pain control.

One of the strengths of this study is that it is, to our knowledge, the first study that provides an analysis of preoperative and sequential follow-up data on quadriceps strength measured using a dynamometer. In contrast to previous studies that base their analyses only on final follow-up session data, we attempted to rule out the influence of physiological muscle strength difference between groups in order to enhance the credibility of the study results. In addition, this is a well-designed randomized, prospective study with a sufficient sample size. Another significance 
of this study is that not only the isometric strength but also the amount of work per unit of time and the extent of recovery were assessed based on the quadriceps force and power measurements, which enabled more accurate evaluation of quadriceps strength after TKA. Furthermore, our study provided quantitative data on the improvement of quadriceps strength after TKA that was achieved irrespective of the type of femoral components.

There are some limitations of this study. First, additional potential advantages of the SR design with regard to mid-flexion range stability and patellofemoral joint were not addressed in the study. This was because the current study was focused on the assessment of quadriceps function recovery, not on the investigation of all benefits of the SR design. In our opinion, however, there is a low likelihood that the theoretical benefit of patellofemoral joint force reduction can be realized in clinical practice, considering that there was no case with anterior knee pain in both groups. The practical importance of such theoretical possibilities should be explored in further research. Another shortcoming of the study is patellar resurfacing was selectively performed. We are unaware of studies that demonstrate the influence of patellar resurfacing in TKA on quadriceps function recovery. Patellar resurfacing vs. non-resurfacing in TKA has been the subject of controversy. However, some meta-analysis studies suggest that patellar resurfacing has no significant clinical implication and reduces the incidence of reoperations related to patellofemoral joint conditions ${ }^{24-27)}$. Therefore, assuming patellar resurfacing was not a factor associated with postoperative quadriceps recovery, we performed the procedure in both groups according to the same criteria adopted by our institution. The incidence of patellar resurfacing was not significantly different between the groups (SR group, 19/55; MR group, 21/54). We believe the influence of patellar resurfacing on quadriceps recovery should be examined further in future research.

\section{Conclusions}

The SR femoral design was not superior to the MR femoral design in terms of quadriceps recovery during the 1-year follow-up after TKA. In addition, the two femoral designs did not result in significant differences with regard to postoperative clinical scores and ROM. Thus, our findings suggest the femoral component design itself would not influence the quadriceps function after TKA.

\section{Conflict of Interest}

No potential conflict of interest relevant to this article was re- ported.

\section{References}

1. Andriacchi TP, Galante JO, Fermier RW. The influence of total knee-replacement design on walking and stair-climbing. J Bone Joint Surg Am. 1982;64:1328-35.

2. Andriacchi TP, Yoder D, Conley A, Rosenberg A, Sum J, Galante JO. Patellofemoral design influences function following total knee arthroplasty. J Arthroplasty. 1997;12:243-9.

3. Kessler O, Durselen L, Banks S, Mannel H, Marin F. Sagittal curvature of total knee replacements predicts in vivo kinematics. Clin Biomech (Bristol, Avon). 2007;22:52-8.

4. Mahoney OM, McClung CD, dela Rosa MA, Schmalzried TP. The effect of total knee arthroplasty design on extensor mechanism function. J Arthroplasty. 2002;17:416-21.

5. Mizner RL, Petterson SC, Stevens JE, Vandenborne K, Snyder-Mackler L. Early quadriceps strength loss after total knee arthroplasty: the contributions of muscle atrophy and failure of voluntary muscle activation. J Bone Joint Surg Am. 2005;87:1047-53.

6. Browne C, Hermida JC, Bergula A, Colwell CW Jr, D’Lima DD. Patellofemoral forces after total knee arthroplasty: effect of extensor moment arm. Knee. 2005;12:81-8.

7. D'Lima DD, Poole C, Chadha H, Hermida JC, Mahar A, Colwell CW Jr. Quadriceps moment arm and quadriceps forces after total knee arthroplasty. Clin Orthop Relat Res. 2001;(392):213-20.

8. Ostermeier S, Stukenborg-Colsman C. Quadriceps force after TKA with femoral single radius. Acta Orthop. 2011; 82:339-43.

9. Jenny JY, Miehlke R, Saragaglia D, Geyer R, Mercier N, Schoenahl JY, Thiel B. Single-radius, multidirectional total knee replacement. Knee Surg Sports Traumatol Arthrosc. 2013;21:2764-9.

10. Stoddard JE, Deehan DJ, Bull AM, McCaskie AW, Amis AA. The kinematics and stability of single-radius versus multiradius femoral components related to mid-range instability after TKA. J Orthop Res. 2013;31:53-8.

11. Cook LE, Klika AK, Szubski CR, Rosneck J, Molloy R, Barsoum WK. Functional outcomes used to compare single radius and multiradius of curvature designs in total knee arthroplasty. J Knee Surg. 2012;25:249-53.

12. Gomez-Barrena E, Fernandez-Garcia C, Fernandez-Bravo A, Cutillas-Ruiz R, Bermejo-Fernandez G. Functional performance with a single-radius femoral design total knee arthro- 
plasty. Clin Orthop Relat Res. 2010;468:1214-20.

13. Hall J, Copp SN, Adelson WS, D’Lima DD, Colwell CW Jr. Extensor mechanism function in single-radius vs multiradius femoral components for total knee arthroplasty. J Arthroplasty. 2008;23:216-9.

14. Palmer J, Sloan K, Clark G. Functional outcomes comparing Triathlon versus Duracon total knee arthroplasty: does the Triathlon outperform its predecessor? Int Orthop. 2014; 38:1375-8.

15. Noble PC, Conditt MA, Cook KF, Mathis KB. The John Insall Award: patient expectations affect satisfaction with total knee arthroplasty. Clin Orthop Relat Res. 2006;452:35-43.

16. Wang H, Simpson KJ, Ferrara MS, Chamnongkich S, Kinsey T, Mahoney OM. Biomechanical differences exhibited during sit-to-stand between total knee arthroplasty designs of varying radii. J Arthroplasty. 2006;21:1193-9.

17. Jo AR, Song EK, Lee KB, Seo HY, Kim SK, Seon JK. A comparison of stability and clinical outcomes in single-radius versus multi-radius femoral design for total knee arthroplasty. J Arthroplasty. 2014;29:2402-6.

18. Ibrahim MS, Alazzawi S, Nizam I, Haddad FS. An evidencebased review of enhanced recovery interventions in knee replacement surgery. Ann R Coll Surg Engl. 2013;95:386-9.

19. Ibrahim MS, Khan MA, Nizam I, Haddad FS. Peri-operative interventions producing better functional outcomes and enhanced recovery following total hip and knee arthroplasty: an evidence-based review. BMC Med. 2013;11:37.

20. Berman AT, Bosacco SJ, Israelite C. Evaluation of total knee arthroplasty using isokinetic testing. Clin Orthop Relat Res.
1991;(271):106-13.

21. Walsh M, Woodhouse LJ, Thomas SG, Finch E. Physical impairments and functional limitations: a comparison of individuals 1 year after total knee arthroplasty with control subjects. Phys Ther. 1998;78:248-58.

22. Berth A, Urbach D, Neumann W, Awiszus F. Strength and voluntary activation of quadriceps femoris muscle in total knee arthroplasty with midvastus and subvastus approaches. J Arthroplasty. 2007;22:83-8.

23. Umrani SP, Cho KY, Kim KI. Patellar eversion does not adversely affect quadriceps recovery following total knee arthroplasty. J Arthroplasty. 2013;28:591-4.

24. Breeman S, Campbell M, Dakin H, Fiddian N, Fitzpatrick R, Grant A, Gray A, Johnston L, Maclennan G, Morris R, Murray D; KAT Trial Group. Patellar resurfacing in total knee replacement: five-year clinical and economic results of a large randomized controlled trial. J Bone Joint Surg Am. 2011;93:1473-81.

25. Chen K, Li G, Fu D, Yuan C, Zhang Q, Cai Z. Patellar resurfacing versus nonresurfacing in total knee arthroplasty: a meta-analysis of randomised controlled trials. Int Orthop. 2013;37:1075-83.

26. Pavlou G, Meyer C, Leonidou A, As-Sultany M, West R, Tsiridis E. Patellar resurfacing in total knee arthroplasty: does design matter? A meta-analysis of 7075 cases. J Bone Joint Surg Am. 2011;93:1301-9.

27. Pilling RW, Moulder E, Allgar V, Messner J, Sun Z, Mohsen A. Patellar resurfacing in primary total knee replacement: a meta-analysis. J Bone Joint Surg Am. 2012;94:2270-8. 